\title{
BUTYRYLCHOLINESTERASE - POSITIVE INNERVATION OF THE SPLEEN IN RATS
}

\author{
Mária Sirotáková1, Katarína Schmidtováa ${ }^{2}$ Monika Kočišová ${ }^{2}$ \\ University P. J. Šafárik, Medical Faculty, Košice, Slovak Republic: Institute of Experimental Medicine ${ }^{1}$, Institute of Anatomy ${ }^{2}$
}

\begin{abstract}
Summary: Innervation of the spleen in rats was studied. Butyrylcholinesterase-(BuChE)-positive nerve components of the organ were visualized by the direct thiocholine method. BuChE-positive nerve components enter the spleen in a common bundle with arteries. In the organ they form characteristic periarterial and periarteriolar plexiform arrangements, which are especially conspicuous around the aa. centrales running through the white pulp. Then, nerve fibres extend away from these plexuses into adjacent layers of trabeculae further into marginal layers of periarterial lymphatic sheath (PALS) as well as into the mantle zone of follicles. Several scattered periarteriolar and solitary nerve fibres can be seen in the marginal sinuses and cords of the red pulp. In the fibrous capsula BuChE-positive nerve fibres can also be seen which have an evident connection with trabecular and parenchymal nerves of the organ. Microscopic findings support the notion that BuChE-positive nerve profiles supply not only the vasculature, but also the parenchymal components of the spleen, and they may participate, to a great extent, in the regulation of the immune processes in this organ.
\end{abstract}

Key words: BuChE-positive innervation; Spleen; Rat

\section{Introduction}

Within the context of developing concept on the immuno-neuro-humoral regulation of vitally important functions of the organism, special attention has been paid to the study of the anatomical base for neuroregulation of the lymphoid organs. Results obtained by more authors indicate significant species-specific feature of the innervation also of the spleen in some mammals. The published data about AChEpositive innervation of the spleen in mammals are somewhat controversial, because more of investigators were unable to found AChE-positive nerve fibres in the parenchyma of the organ $(8,15,16)$, while the others described only a small density of nerve fibres near the hilus (7). On the base of a more detailed analysis of the publish data on the adrenergic innervation of the spleen in some mammals $(1,5,6)$ as well as on the results of our previous work on the study of nerve supply of primary lymphoid organs in various mammals $(9,10,17,18)$, we have examined butyrylcholinesterase (BuChE)-positive innervation of the spleen in rats, because the published data about BuChE-positive nerve supply of this organ in rats are still lacking.

\section{Material and methods}

Clinically healthy adult animals of both sexes were used in the study. The spleen of 25 rats (Wistar, weighing 250-350 g) were examined. Before investigation, the ani- mals were kept in the Central Animal Husbandry quarters under veterinary care (food and drinking water were supplied ad libitum). The directions of the Council of European Communities (86/609/EEC) on animal care have been maintained. Handling of experimental animals was performed under the supervision of the Ethical Commitee of the Medical Faculty of P. J. Šafárik University. The animals were anesthetized with pentobarbital $(40 \mathrm{mg} / \mathrm{kg}$ i.p.). BuChE-positive nerve profiles were demonstrated by means of the direct thiocholine method of cytochemical evidence of AChE (3). Tissue blocks were fixed in $4 \%$ paraformaldehyde (pH 7,4) at $4{ }^{\circ} \mathrm{C}$ for 2-4 h. Sections (16-20 $\mu$ m thick) were cut with a cryostat, incubated in the incubation medium $(\mathrm{pH} \mathrm{5,5)}$ for 2-4 h. Individual sections were mounted on glass slides. Control sections were incubated as follows: (1) incubation in a medium free of BW 284 c 51 (Sigma, selective inhibitor of specific AChE), (2) incubation in a medium containing acetylthiocholine iodide and iso-OMPA (Sigma, selective inhibitor of nonspecific esterases). Both the microscopic examination and photographic documentation were performed using a Jenalumar 2 microscope (Zeiss, JenaGermany).

\section{Results}

BuChE-positive nerve components enter the spleen in a common bundle with a. lienalis and its branches. Then, in the organ they pass together with arteries in the form of typi- 


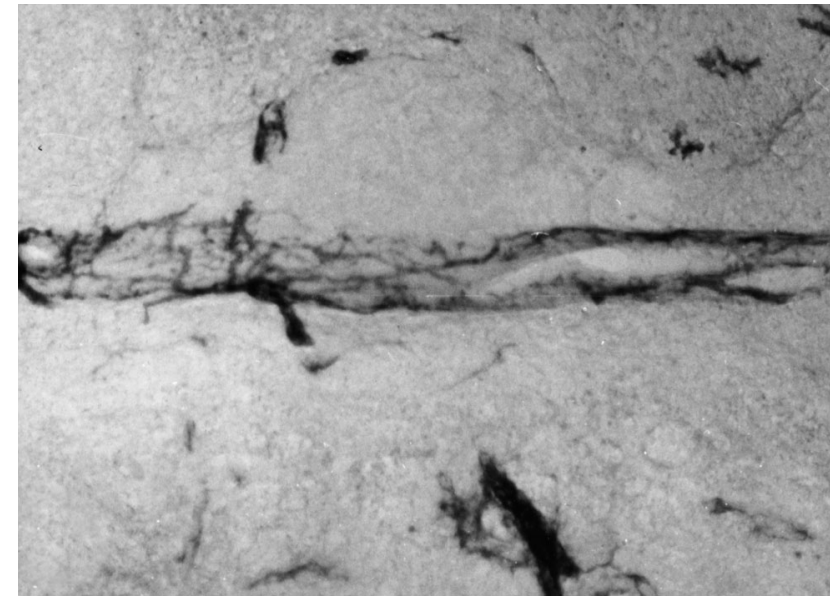

Fig. 1a: Plexiform arrangement of nerve fibres around the arteriolae running in the septum. Magn. x 250.

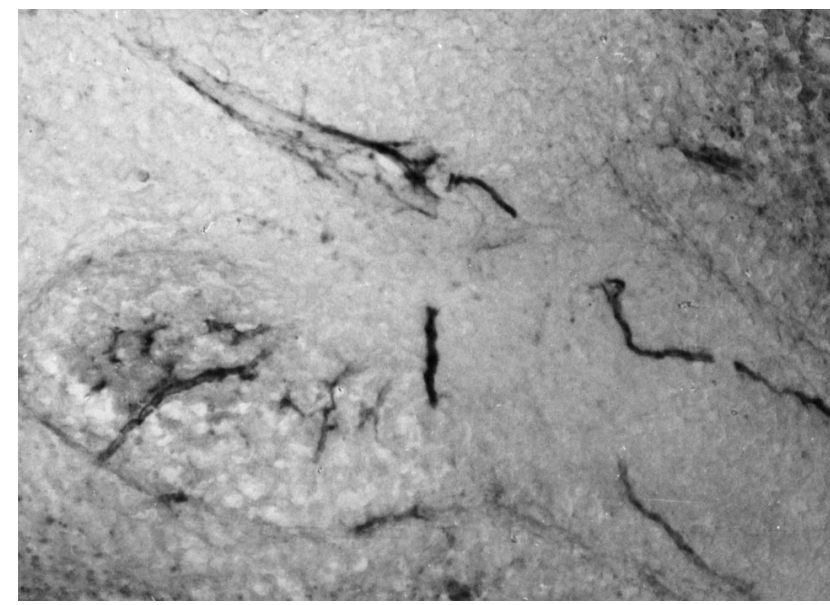

Fig. 2: Individual thicker nerve profiles and adventitial plexiform nerve formations are lying in the vicinity of the lymphatic follicle. Very fine linear BuChE-positive non-neural profiles may be seen also as crossing the follicle. Magn. X 250

cal carrying periarterial and also more delicate plexuses, consisting mainly of preterminal and terminal fibres (Figs. 1a,b).

These fine plexuses lie in close contact with the external side of muscular media layer, sometimes called "adventitial“ plexuses. Relatively dense plexuses with abundant amount of reaction product also accompany the aa. centrales running through the white pulp. The main perivascular nerve profiles were distributed in the fibrous trabeculae as well as in the periarteriolar lymphatic sheath (PALS). Some BuChE-positive nerve fibres also pass through the trabeculae and capsule of the organ without apparent connection with vascular branches. In the thicker fibrous trabeculae, BuChE-positive nerve fibres run more or less parallel to the adjacent artery or arteriole, mostly as isolated and sometimes, also branching nerve profiles (Fig. 2).

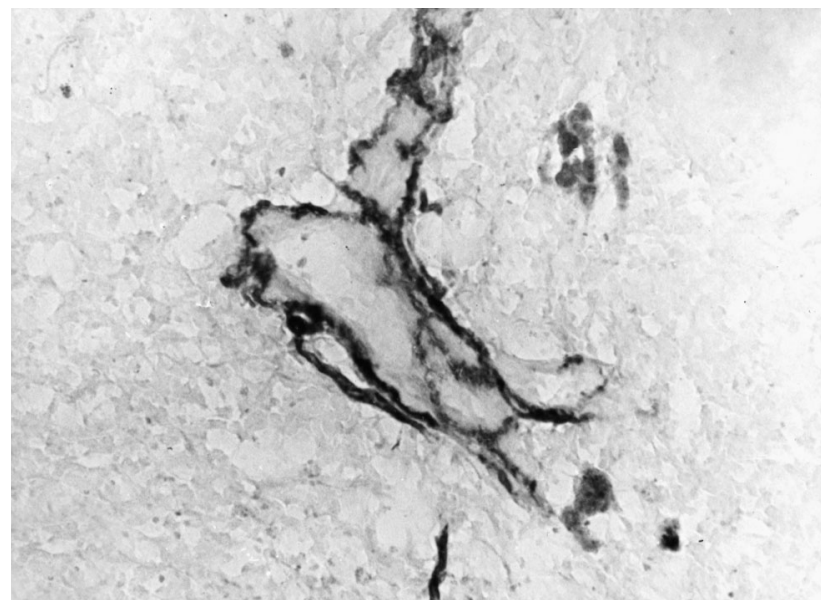

Fig. 1b: Intensive BuChE-positive nerve profiles around the wall of the arteriolae and its branches. Magn. x 500 .

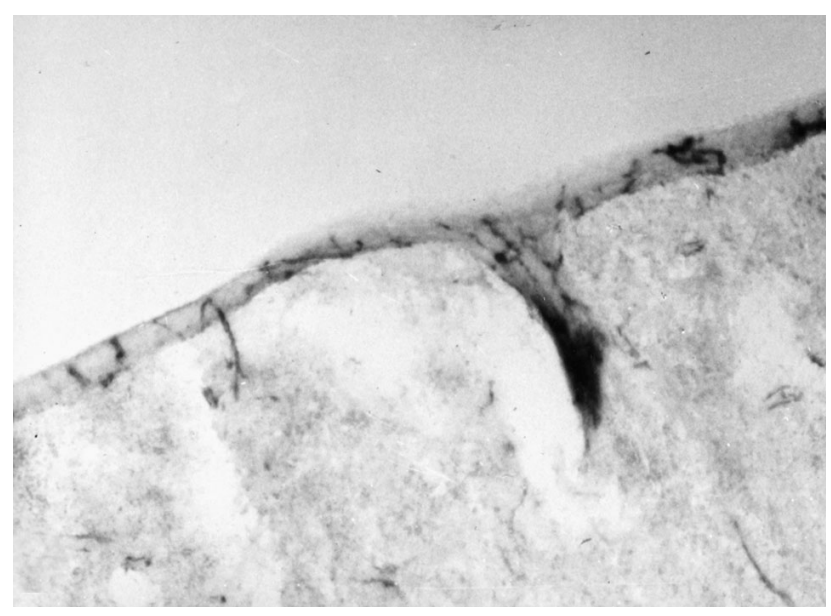

Fig. 3: BuChE-positive nerve fibres in the fibrous capsula and trabeculae of the rat spleen. Magn. x 250.

Within PALS they can be seen most often in the marginal zone between the red and white pulp. BuChE-positive delicate nerve profiles, with small amount of reaction coloured product, were also recorded in the mantle zone of lymphatic follicles. However, as a rule, they do not penetrate into this formation. While the BuChE-positive innervation of the white pulp within the PALS is somewhat poorer, the occurence of periarterial or periarteriolar and individual nerve profiles on the bordeline of the red and white pulp is evident. In addition, the presence of several scattered periarteriolar and solitary nerve fibres was also recorded in the cords or in the wall of the red pulp sinuses.

The wall of large and larger splenic veins was innervated poorly and partially. BuChE-positive periarterial and individual nerve fibres also occur in the fibrous capsula of 
the organ (Fig. 3). Their connection with perivascular structure or parenchyma, was mostly quite evident.

\section{Discussion}

BuChE-positive innervation of the spleen in rats is marked by the more common characteristics with the adrenergic supply of this organ in other small mammals $(5,6,19)$ and BuChE-positive innervation in rabbits (20) and concerning the fact that these nerves enter the organ together with arteries round which they form very conspicuous and typical thicker nerve plexiform formation that fulfil the role of a carrying substrates for the peripheral neuro-effector actions.

It is well known that all the macro- and microscopic structural characteristics of various components of the organism are formed for certain functions. Like in, the spleen is a filter for different non-immunogenic particles of organic or inorganic origin as well as functions of the microenvironment for balanced immune processes. Moreover, it also has the function of a blood reservoir or organ participating, to a great extent, in the regulation of blood re-distribution in the organism (4).

Reactions of smooth muscle cells to the released neurotransmitters both in the vasal media on the arterial and venous sides of the bloodstream as well as in fibrous trabeculae and capsula are among the factors that markedly participate in the regulation of blood flow. In rats, similarly as in rabbits, BuChE-positive nerve components with high amount of coloured reaction product, are found around central artery and its branches in the white pulp, or in close proximity of vascular media of arterial branches, running through in the fibrous trabeculae. Further occurence of BuChE-positive nerve profiles has also been recorded on the bordeline of the red and white pulps (14). There are authors $(5,13,21)$ who suggest that released neurotransmitter may influence the functions of the prarenchyma - entering T-and B-lymphocytes as well processes of the antigen uptage by dentritic cells and macrophages. Subsequent activation of T-lymphocytes could be influenced mainly within the range of the PALS borderline layer, whereas the activation of B-lymphocytes takes place mainly in PALS perifollicular and only partially in the marginal zone. The neurotransmitter may influence also the entry of lymphocytes into thin-wall initial compartments of the venous bed $(11,12,21)$.

Nerve fibres in T-cell-dependent areas are in close vicinity or in contact with migrating lymphocytes, plasma cells, macrophages, and mast cells, but also with arterioles where they participate in local modulation of humoral and cellular responses of the organism and indirectly in overall immunological responses (5).

Our findings of BuChE-positive nerve fibres in the red pulp are in contrast with the data of other authors $(2,17,18)$ who suggest that AChE-positive nerve fibres in spleen of rats reached only the border of the white and red pulp, and they do not enter the red pulp. Similarly these authors have not found any AChE-positive periarteriolar or individual nerve fibres in the fibrous capsula of the organ.

Based upon our findings, it can be stated that the patterns of BuChE-positive innervation of the spleen in rats agree in principle with those in rabbits. Only the total number of BuChE-positive nerve profiles, supplying larger vessels branches, and their occurence in the perifollicular topography, are in rabbits apparently higher (20).

Our findings are in agreement with those found by the authors who state that nerve components supply not only the vasculature, but also the parenchyma of lymphoid organs with specific functional compartments and do not enter their B-cell compartments.

\section{Conclusion}

On the basis of distribution patterns of BuChE-positive nerve profiles in the spleen of rats it can be concluded that besides regulation of vasomotor activity, direct or indirect effects of released neurotransmitters on immunocompetent cells cannot be excluded.

\section{References}

1. Bellinger DL, Felten SY, Collier TJ, Felten DL. Noradrenergic sympathetic in nervation of the spleen: IV. Morphometric analysis in adult and aged F344 rats. J Neurosci Res 1987; 18:55-63.

2. Bellinger DL, Lorton D, Hamil RW, Felten SY, Felten DL. Acetylcholinesterase staining and choline acetyltransferase activity in young adult rat spleen. Lack of evidence for cholinergic innervation. Brain Behav Immun 1993; 7:191-204.

3. El Badawi A, Schenk EA. Histochemical methods for separate consecutive and simultaneous demonstration of acetylcholinesterases and norepinephrine in cryostat sections. J Histochem Cytochem 1967;15:580-8.

4. Felten DL. Direct innervation of lymphoid organs: Substrate for neurotransmitter signaling cells of the immune system. Neuropsychobiology 1993;28:110-2.

5. Felten DL, Ackerman KD, Wiegand SJ, Felten SY. Noradrenergic sympathetic in nervation of the spleen: Nerve fibres spleen associate with lymphocytes and macrophages in specific compartments of the splenic white pulp. J Neurosci Res 1987; 18:28-36.

6. Felten SY, Olschowka J. Noradrenergic sympathetic innervation of the spleen: II Tyrosine hydroxylase (TH)-positive nerve terminals form synaptic-like contacts in the splenic white pulp. J Neurosci Res 1987;18:37-48.

7. Filenz M. The innervation of the cat spleen. Proc R Soc London 1970;174: 459-68.

8. Heusermann U, Stutte HJ. Electron microscopic studies of the innervation of the human spleen. Cell Tiss Res 1977;184:225-36.

9. Kočišová M. Presence of butyrylcholinesterase (BuChE)-positive nerve structures in the thymus of rat. Collection of scientific work from 4. Morphological Day, Košice, 2001;41-2.

10. Kočišová M, Sirotáková M, Schmidtová K, Dorko F, Rybárová S. Functional morphology of autonomous innervation of the bursa cloacalis in birds. Acta Med Martiniana 2002;2/1:3-6.

11. Madden KS, Felten SY, Felten DL, Sundaresan PR, Livnat S. Sympathetic nervous system modulation of the immune system. Brain Behav Immun 1989;3: $72-81$.

12. Madden KS, Moynihan JA, Brenner GJ, Felten SY, Felten DL, Livnat S. Sympathetic nervous system modulation of the immune system. III. Alteration in $\mathrm{T}$ and $\mathrm{B}$ cell proliferation and differentiation in vitro following chemical sympathectomy. J Immunol 1994:49:77-8.

13. Mignini F, Streccioni V, Amenta F. Autonomic innervation of immune organs and neuroimmune modulation. Autonom Autacoid Pharmacol 2003;23:1-25.

14. Pellas TC, Weis L. Deep splenic lymphatic vessels in the mouse. A route of splenic exit for recirculating sheep lymphocytes. Am J Anat 1990;187:347-54.

15. Reily FD. Innervation and vascular pharmacodynamics of the mammalian spleen. Experientia 1985;41:187-95.

16. Reily FC, Mc Cuskey RS, Neincke HA. Studies on the hemopoietic microenviroment. VIII. Adrenergic and cholinergic innervation of the murine spleen. Anat Rec 1976;185:109-29. 
17. Schmidtová K, Bánovská E, Kočišová M, Gomboš A. Effect of irradiation on distribution of acetylcholinesterase (AChE)-positive nerve fibres in the spleen of rats. Func Develop Morphol 1994;4:261-2.

18. Schmidtová K, Bánovská E, Miklošová M. Development and distribution of acetylcholinesterase (AChE)-positive nerve fibres in the spleen of rats and chickens. Folia Vet 1995;39:75-9

19. Sirotáková M, Kočišová M, Schmidtová K, Dorko F, Danko J. Functional morphology of adrenergic innervation of guinea pig spleen. Acta Vet Brno 2002; 71:159-62.

20. Sirotáková M, Maretta M, Marettová E. Butyrylcholinesterase-positive innervation of the spleen in rabbits. New trends in the Morphology. Collection of scientific works of Jessenius Faculty of Medicine, Martin, July 3-5, 2001;184-5 (in Slovak).

21. Tayebati SK, El-Assouad D, Ricci A, Amenta F. Immunochemical and immunocytochemical characterization of cholinergic markers in human peripheral blood lymphocytes. J Neuroimmunol 2002;132:147-55.

Submitted March 2004.

Accepted June 2004.

MVDr. Mária Sirotáková, CSc.,

P. J. Šafárik University, Medical Faculty,

Institute of Experimental Medicine, Tr. SNP, 04011 Košice 1, Slovak Republic. 